\title{
Accurate Frequency Estimation for Real Harmonic Sinusoids
}

\author{
K. W. Chan and H. C. So, Member, IEEE
}

\begin{abstract}
A linear prediction based method is proposed for real harmonic sinusoidal frequency estimation. The estimator basically involves two steps. An initial fundamental frequency estimate is first obtained by solving a standard least-squares equation with exploitation of the harmonic structure of the sinusoidal signal or by using the MUSIC approach. Based on the initial estimate, an optimally weighted least squares cost function is then constructed from which the final estimate is acquired. Computer simulations show that the performance of the estimator approaches Cramér-Rao lower bound for sufficiently high signal-to-noise ratios and/or data lengths.
\end{abstract}

Index Terms-Frequency estimation, harmonic sinusoidal signals, weighted least squares.

\section{INTRODUCTION}

A LTHOUGH there are numerous algorithms for frequency estimation of sinusoidal signals in the literature such as maximum likelihood [1], nonlinear least squares [2] and subspace methods [3]-[5], little attention [6], [7] has been paid to the special case of harmonic sinusoids. In fact, harmonic frequency estimation has important applications in speech signal processing [8]-[10], automotive control systems [11] as well as instrumentation and measurement [12].

In [6], complex harmonics are considered and accurate frequency estimation is achieved via weighted least squares (WLS) where the weighting matrix is given by the Markov estimate [13]. To construct the Markov estimate, initial estimation of all tone amplitudes, frequencies and phases is required, which is fulfilled by applying the MUSIC [4] approach. In this paper, we extend the idea of the WLS technique to real harmonic sinusoidal frequency via utilizing the linear prediction (LP) property as well as the harmonic structure. Unlike [6], the proposed algorithm directly estimates the fundamental frequency and does not involve computation of the sinusoidal amplitudes and phases, which is not our concern.

The rest of the paper is organized as follows. Algorithm development for real harmonic sinusoidal frequency estimation is presented in Section II. In Section III, simulation results are included to evaluate the performance of the estimator in different conditions. Finally, conclusions are drawn in Section IV.

Manuscript received September 30, 2003; revised November 28, 2003. The associate editor coordinating the review of this manuscript and approving it for publication was Dr. Vitor H. Nascimento.

The authors are with the Department of Computer Engineering and Information Technology, City University of Hong Kong, Kowloon, Hong Kong (e-mail frankie.chan@student.cityu.edu.hk; ithcso@cityu.edu.hk).

Digital Object Identifier 10.1109/LSP.2004.830115

\section{PROPOSED AlgORIthm}

The harmonic sinusoidal signal model is

$$
x_{n}=s_{n}+q_{n} \quad n=0,1, \ldots, N-1
$$

where

$$
s_{n}=\sum_{m=1}^{M} A_{m} \cos \left(m \omega n+\phi_{m}\right)
$$

where $\omega, A_{m}$ and $\phi_{m}$ are unknown constants which represent the fundamental frequency, the $m$ th amplitude and phase corresponding to the $(m-1)$ th harmonic, respectively, and $q_{n}$ is a white additive noise with unknown variance $\sigma^{2}$. The aim is to estimate $\omega$ from the $N$ samples of $x_{n}$. Notice that when the number of sinusoids, namely, $M$, is not available, it can be accurately estimated using the Akaike information criterion [14], minimum description length [15], or more recent techniques including singular value decomposition [16] and eigenvalue factorization [17]. As a result, we reasonably assume that $M$ is known a priori in this paper as our focus is in frequency estimation.

Starting with the LP property of sinusoidal signals, it is well known that $s_{n}$ is perfectly predictable from its past $2 M$ sampled values as

$$
\mathbf{s}_{n}^{T} \mathbf{a}=0
$$

where

$$
\begin{aligned}
\mathbf{s}_{n}= & {\left[s_{n}+s_{n-2 M}, s_{n-1}+s_{n-2 M+1}, \ldots,\right.} \\
& \left.s_{n-M+1}+s_{n-M-1}, s_{n-M}\right]^{T}, \\
n \geq & 2 M \\
\mathbf{a}= & {\left[a_{0}, a_{1}, \ldots, a_{M}\right]^{T}, \quad a_{0}=1 }
\end{aligned}
$$

and $T$ represents the transpose operation. The frequency information is uniquely related to the LP coefficients in a and it is noteworthy that we have exploited the symmetry of $\left\{a_{i}\right\}$ [18], namely, $a_{i}=a_{2 M-i}, i=0,1, \ldots, M-1$. More exactly, the harmonic frequencies are given by the phases of the roots of

$$
\begin{aligned}
f(z) & =\prod_{m=1}^{M}\left(z^{2}-2 z \cos (m \omega)+1\right) \\
& =a_{M} z^{M}+\sum_{n=0}^{M-1} a_{n}\left(z^{n}+z^{2 M-n}\right)=0 .
\end{aligned}
$$

The next step is to express $\cos (m \omega), m=2,3, \ldots, M$, in terms of $\cos (\omega)$, which is achieved by using the Chebyshev polynomial of the first kind [19]

$$
\cos (n \omega)=T_{n}(\cos (\omega))
$$


where

$$
T_{n}(x)=\frac{n}{2} \sum_{r=0}^{\lfloor n / 2\rfloor} \frac{(-1)^{r}}{n-r} C_{r}^{n-r}(2 x)^{n-2 r}
$$

and $\lfloor\alpha\rfloor$ denotes rounding $\alpha$ to the nearest integer towards minus infinity. Substituting (4) into (3), a can be written as $\mathbf{a}=\mathbf{P y}$ where $\mathbf{P} \in \mathcal{R}^{(M+1) \times(2 M+1)}$ and $\mathbf{y}=\left[\cos ^{2 M}(\omega), \cos ^{2 M-1}(\omega), \ldots, 1\right]^{T}$. The corresponding LP error function is then

$$
e_{n}=\mathbf{x}_{n}^{T} \hat{\mathbf{a}}=\mathbf{x}_{n}^{T} \mathbf{P} \hat{\mathbf{y}}, \quad n=2 M, 2 M+1, \ldots, N-1
$$

where

$$
\begin{aligned}
\mathbf{x}_{n}=\left[x_{n}+x_{n-2 M}, x_{n-1}+\right. & x_{n-2 M+1}, \ldots, \\
& \left.x_{n-M+1}+x_{n-M-1}, x_{n-M}\right]^{T}
\end{aligned}
$$

and $\hat{\mathbf{a}}$ is an estimate of a with $\hat{a}_{0}=1$ and $\hat{\mathbf{y}}$ is a function of the frequency estimate $\hat{\omega}$. Representing all the $(N-2 M)$ prediction errors in vector form, we have

$$
\mathbf{e}=\mathbf{X P} \hat{\mathbf{y}}
$$

where

$$
\mathbf{X}=\left[\mathbf{x}_{N-1}, \mathbf{x}_{N-2}, \ldots, \mathbf{x}_{2 M}\right]^{T} .
$$

Since it is well known that minimizing the LP errors via standard least squares [20] will give biased frequency estimates, we propose to use WLS with an optimal weighting matrix which results in unbiased frequency estimation. Based on the WLS technique, the fundamental frequency is estimated via minimization of the following cost function

$$
J(\hat{\omega})=\mathbf{e}^{T} \mathbf{W e}
$$

where $\mathbf{W}$ is a symmetric weighing matrix. The optimal $\mathbf{W}$ is given by the Markov estimate and has the form of

$$
\mathbf{W}=\sigma^{2}\left[E\left\{\mathbf{e e}^{T}\right\}\right]^{-1}
$$

where $\mathbf{e}$ is constructed using the ideal $\omega, E$ denotes expectation and ${ }^{-1}$ is the matrix inverse operation. It has been shown (see the Appendix) that the optimal $\mathbf{W}^{-1}$ is a Toeplitz matrix and thus it is characterized by its first column, say, $\mathbf{v}$

$$
\mathbf{v}=\left[\begin{array}{ll}
\mathbf{u}^{T} & \mathbf{0}_{1 \times(N-4 M-1)}
\end{array}\right]^{T}
$$

where

$$
\begin{aligned}
\mathbf{u} & =\mathbf{I}_{2 M+1} \otimes\left(\mathbf{y}^{T} \mathbf{P}^{T}\left[\begin{array}{ll}
\mathbf{I}_{M+1} & \mathbf{L}^{T}
\end{array}\right]\right) \\
& \cdot\left[\begin{array}{c}
\mathbf{K}_{2 M+1,0} \\
\vdots \\
\mathbf{K}_{2 M+1,2 M}
\end{array}\right]\left[\begin{array}{c}
\mathbf{I}_{M+1} \\
\mathbf{L}
\end{array}\right] \mathbf{P y} \\
\mathbf{L} & =\left[\begin{array}{ccccc}
0 & 0 & \ldots & 1 & 0 \\
0 & \vdots & \ldots & 0 & 0 \\
\vdots & 1 & \ldots & 0 & 0 \\
1 & 0 & \ldots & 0 & 0
\end{array}\right]
\end{aligned}
$$

is of dimension $M \times(M+1)$ and $\mathbf{0}_{i \times j}$ is a zero matrix with dimension $i \times j, \otimes$ denotes Kronecker matrix product, $\mathbf{I}_{i}$ represents the identity matrix with dimension $i \times i$ and $\mathbf{K}_{i, j} \in \mathcal{R}^{i \times i}$ has all elements 0 except 1 in its lower $j$ th diagonal. In practice,
W should be constructed from the frequency estimate, which is obtained from solving

$$
\begin{aligned}
\frac{\partial J(\hat{\omega})}{\partial \cos (\omega)}=0 \Rightarrow \operatorname{tr}\left(\mathbf{W} \frac{\partial\left(\mathbf{e e}^{T}\right)}{\partial \cos (\omega)}\right) & =0 \\
\Rightarrow \operatorname{tr}\left(\frac{\partial\left(\hat{\mathbf{y}} \hat{\mathbf{y}}^{T}\right)}{\partial \cos (\omega)} \mathbf{P}^{T} \mathbf{X}^{T} \mathbf{W X P}\right) & =0
\end{aligned}
$$

where tr denotes trace operation. Since all elements of $\partial\left(\hat{\mathbf{y}} \hat{\mathbf{y}}^{T}\right) / \partial \cos (\omega)$ are identical in every off-diagonal, the coefficients of the polynomial can be easily determined by summing the off-diagonal elements of $\mathbf{P}^{T} \mathbf{X}^{T} \mathbf{W X P}$. The fundamental frequency estimate is chosen as the root which has the smallest value when substituting into $J(\hat{\omega})$. Since $\mathbf{W}$ depends on $\hat{\mathbf{y}}$ which is not available at the beginning, we suggest the following iterative procedure.

i) Find an initial fundamental frequency estimate. This is achieved by setting $\mathbf{W}=\mathbf{I}_{N-2 M}$, finding all the roots in (10) and then choosing the one which minimizes (7) as $\hat{\omega}$, or using the MUSIC algorithm for real sinusoids [21] where $\hat{\omega}$ equals the smallest frequency estimate. We refer the algorithms using the first and second initializations to as the WLS and WLS-MUSIC methods, respectively.

ii) Use $\hat{\omega}$ to construct $\mathbf{W}$ based on (8).

iii) Find all the roots in (10) and choose the one which minimizes (7) as $\hat{\omega}$.

iv) Repeat ii) and iii) until the absolute difference between successive fundamental frequency estimates is less than $\delta$, where $\delta$ is a small positive constant.

It is noteworthy that as in many iterative frequency estimation algorithms [22], [23], there is no guarantee of convergence for the proposed method, although extensive simulation results have been performed to illustrate its global convergence for sufficiently large signal-to-noise ratio (SNRs) and/or data lengths.

\section{NUMERICAL EXAMPLES}

Simulation tests have been carried out to evaluate the performance of the proposed harmonic frequency estimator by comparing with the MUSIC approach for real sinusoids [21] as well as Cramér-Rao lower bound (CRLB). Since [21] does not assume harmonically related frequencies, a better fundamental frequency estimate is simply to use a standard least squares procedure on all frequency estimates and the performance of this improved estimator, which is referred to as the modified MUSIC, is also included. The signal consists of $M=3$ harmonic components corrupted by a zero-mean white Gaussian noise. The amplitudes of the real sinusoids are all equal to 1 while the phases for the fundamental tone, first and second harmonics are $0, \pi / 3$ and $\pi / 4$, respectively. The parameter of the termination criterion in the proposed approach is given by $\delta=10^{-3}$, and in most realizations at most three iterations are needed for the algorithm convergence. All results provided are averages of 500 independent runs.

In the first example, the mean-square frequency errors (MSFEs) of the proposed algorithm versus SNR are investigated. The number of samples $N$ is set to 20 and the funda- 


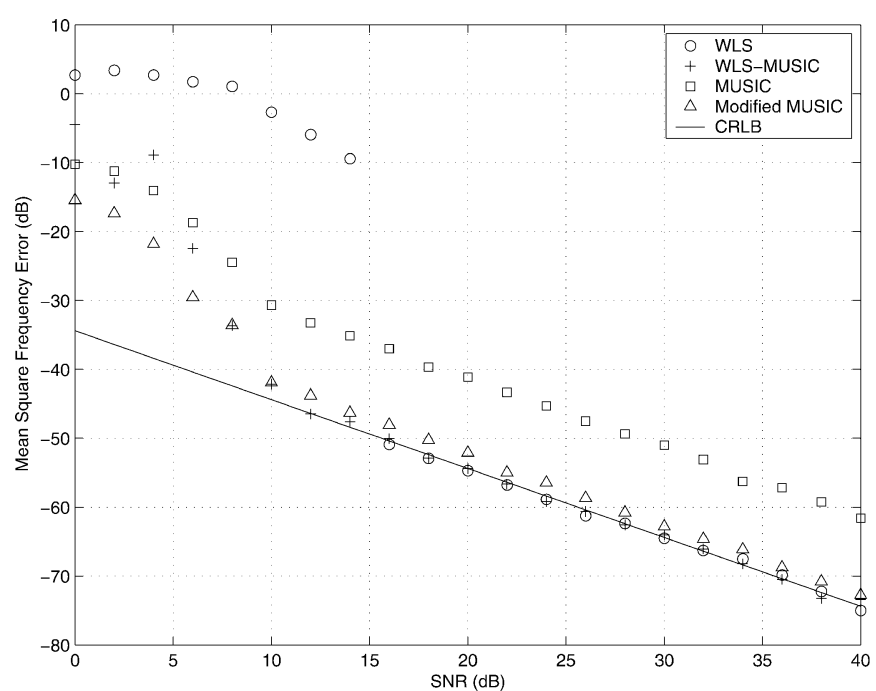

Fig. 1. Mean square frequency errors versus SNR at $\omega=0.2 \pi$ and $N=20$.

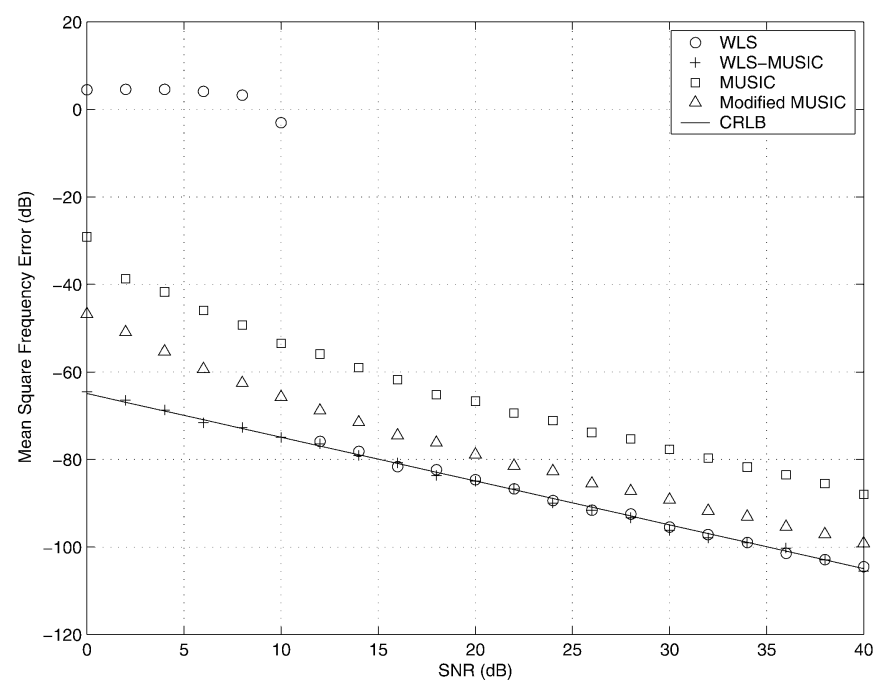

Fig. 2. Mean square frequency errors versus SNR at $\omega=0.2 \pi$ and $N=200$.

mental frequency $\omega$ is selected as $0.2 \pi$ while different SNRs are obtained by properly scaling the noise sequence. In Fig. 1, we see that the MSFEs of the WLS and WLS-MUSIC algorithms attain the CRLB for SNR $\geq 16 \mathrm{~dB}$ and $\mathrm{SNR} \geq 12 \mathrm{~dB}$, respectively. It is because the initial estimates provided by the WLS method are biased while those of the WLS-MUSIC algorithm are unbiased, which makes the former more probably converge to local instead of global solution for smaller SNR conditions. Apart from the threshold regions, the proposed approach outperforms the MUSIC and modified MUSIC by approximately $13 \mathrm{~dB}$ and $2 \mathrm{~dB}$, respectively. As a result, the optimality of the WLS scheme is demonstrated when the sample size is small.

The above test is repeated for $N=200$ and the results are shown in Fig. 2. Comparing with Fig. 1, similar results are observed except that the threshold SNRs of all methods are reduced. The optimality of the WLS scheme for large data lengths is thus demonstrated.

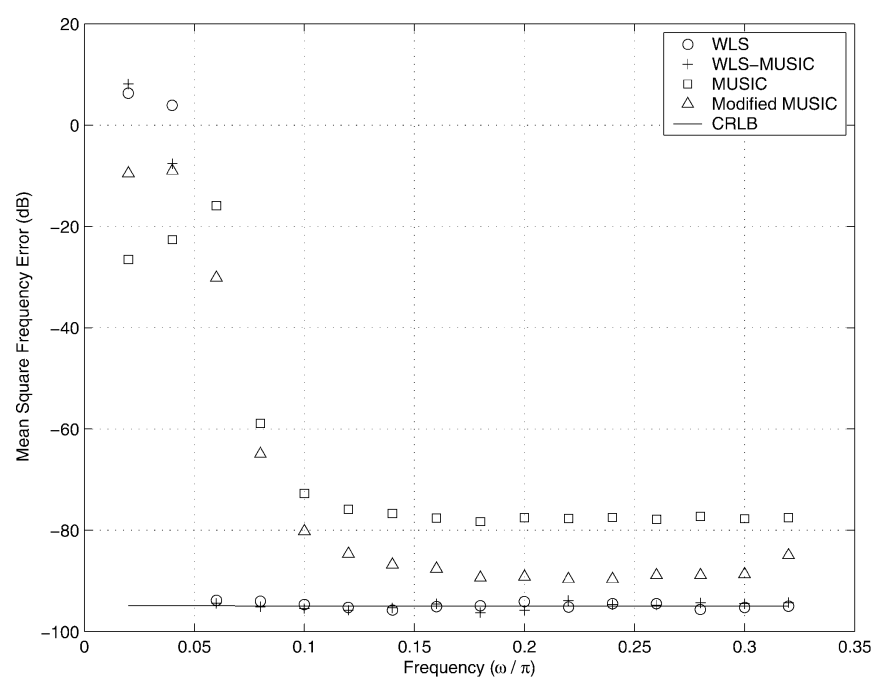

Fig. 3. Mean square frequency errors versus $\omega$ at $N=200$ and SNR $=$ $30 \mathrm{~dB}$.

In the final example, the MSFEs of all methods versus different $\omega$ is studied. Fig. 3 shows the results when the SNR is kept as $30 \mathrm{~dB}, N=200$, and the fundamental frequency is varied in the range of $[0.02,0.32] \pi$. We observe that when the harmonic frequencies are sufficiently separated, say, $\omega \geq$ $0.06 \pi$, the MSFEs attain the CRLB. Furthermore, the superiority of the WLS approach over the MUSIC algorithms is again observed.

\section{CONCLUSIONS}

We have presented an iterative weighted least squares approach for real harmonic sinusoidal frequency estimation. Through computer simulations, it is shown that the mean square frequency errors of the developed method can attain Cramér-Rao lower bound for sufficiently high signal-to-noise ratios and/or data lengths. Comparing with other optimal estimators such as maximum likelihood estimation and nonlinear least squares, the proposed estimator is computationally efficient because no searching procedure is involved.

\section{APPENDIX}

Using the ideal $\omega$, the inverse of $\mathbf{W}$ is derived as

$$
\begin{aligned}
\mathbf{W}^{-1} & =\frac{E\left\{\mathbf{e e}^{T}\right\}}{\sigma^{2}} \\
& =\frac{E\left\{(\mathbf{X P y})(\mathbf{X P y})^{T}\right\}}{\sigma^{2}} \\
& =\frac{E\left\{(\mathbf{S}+\mathbf{Q})(\mathbf{P y})(\mathbf{P y})^{T}(\mathbf{S}+\mathbf{Q})^{T}\right\}}{\sigma^{2}} \\
& =\frac{E\left\{\mathbf{S}(\mathbf{P y})(\mathbf{P y})^{T} \mathbf{S}^{T}\right\}+E\left\{\mathbf{Q}(\mathbf{P y})(\mathbf{P y})^{T} \mathbf{Q}^{T}\right\}}{\sigma^{2}} \\
& =\frac{E\left\{\mathbf{Q}(\mathbf{P y})(\mathbf{P y})^{T} \mathbf{Q}^{T}\right\}}{\sigma^{2}} .
\end{aligned}
$$


It is easy to show that $\mathbf{W}^{-1}$ is symmetric Toeplitz and thus only the first column of $\mathbf{W}^{-1}$, say, $\mathbf{v}$, is needed to be determined. Denoting by $\mathbf{q}_{i}$ the $i$ th row of $\mathbf{Q}$, we have

$$
\begin{aligned}
\mathbf{v} & =E\left\{(\mathbf{P y})^{T} \mathbf{q}_{1}^{T} \mathbf{Q P y}\right\} \\
& =E\left\{\mathbf{Q} \otimes\left((\mathbf{P y})^{T} \mathbf{q}_{1}^{T}\right) \mathbf{P y}\right\} \\
& =\left(\mathbf{I}_{N-2 M} \otimes(\mathbf{P y})^{T}\right) E\left\{\mathbf{Q} \otimes \mathbf{q}_{1}^{T}\right\} \mathbf{P y} .
\end{aligned}
$$

Let us first consider the term $\mathbf{H}=E\left\{\mathbf{Q} \otimes \mathbf{q}_{1}^{T}\right\}$. The $i$ th row, $i=1,2, \ldots, N-2 M$, of $\mathbf{Q}$ is given by

$$
\left[q_{N-1}, q_{N-2}, \ldots, q_{0}\right] \mathbf{K}_{N, i-1} \mathbf{R}
$$

where

$$
\mathbf{R}=\left[\begin{array}{c}
\mathbf{I}_{M+1} \\
\mathbf{L} \\
\mathbf{0}_{(N-2 M-1) \times(M+1)}
\end{array}\right]
$$

Since $\mathbf{q}_{1}^{T}=\left(\left[q_{N-1}, \ldots, q_{0}\right] \mathbf{K}_{N, 0} \mathbf{R}\right)^{T}=$ $\left(\left[q_{N-1}, \ldots, q_{0}\right] \mathbf{R}\right)^{T}$, the expected value of the Kronecker product of the $i$ th row of $\mathbf{Q}$, namely, $\mathbf{q}_{i}$, and $\mathbf{q}_{1}$, after vectorization, is

$$
\begin{array}{r}
E\left\{\operatorname { v e c } \left(\left(\left[q_{N-1}, \ldots, q_{0}\right] \mathbf{K}_{N, i-1} \mathbf{R}\right)\right.\right. \\
\left.\left.\otimes\left(\left[q_{N-1}, \ldots, q_{0}\right] \mathbf{R}\right)^{T}\right)\right\}
\end{array}
$$

where vec represents vectorization operation. Expression (14) can also be written as

$$
\begin{aligned}
E & \left\{\left(\mathbf{R}^{T} \mathbf{K}_{N, i-1}^{T}\left[q_{N}, \ldots, q_{0}\right]^{T}\right) \otimes\left(\mathbf{R}^{T}\left[q_{N}, \ldots, q_{0}\right]^{T}\right)\right\} \\
= & E\left\{\left(\mathbf{R}^{T} \mathbf{K}_{N, i-1}^{T} \otimes \mathbf{R}^{T}\right)\right. \\
& \left.\cdot\left(\left[q_{N}, \ldots, q_{0}\right]^{T} \otimes\left[q_{N}, \ldots, q_{0}\right]^{T}\right)\right\} \\
= & \left(\mathbf{R}^{T} \mathbf{K}_{N, i-1}^{T} \otimes \mathbf{R}^{T}\right) \operatorname{vec}\left(\mathbf{I}_{N}\right) \sigma^{2} \\
= & \operatorname{vec}\left(\mathbf{R}^{T} \mathbf{K}_{N, i-1} \mathbf{R}\right) \sigma^{2} \\
= & \operatorname{vec}\left(\left[\begin{array}{c}
\mathbf{I}_{M+1} \\
\mathbf{L}
\end{array}\right]^{T} \mathbf{K}_{2 M+1, i-1}\left[\begin{array}{c}
\mathbf{I}_{M+1} \\
\mathbf{L}
\end{array}\right]\right) \sigma^{2} .
\end{aligned}
$$

Since the dimensions of the matrices in (14) and (15) are the same, the vec operator can be removed and we get

$$
\begin{aligned}
& \left(\left[q_{N-1}, \ldots, q_{0}\right] \mathbf{K}_{N, i-1} \mathbf{R}\right) \otimes\left(\left[q_{N-1}, \ldots, q_{0}\right] \mathbf{R}\right)^{T} \\
& =\left[\begin{array}{c}
\mathbf{I}_{M+1} \\
\mathbf{L}
\end{array}\right]^{T} \mathbf{K}_{2 M+1, i-1}\left[\begin{array}{c}
\mathbf{I}_{M+1} \\
\mathbf{L}
\end{array}\right] \sigma^{2} .
\end{aligned}
$$

Grouping the results in (16) for all rows, $i=1,2, \ldots, N-2 M$, yields $\mathbf{H}$

$$
\begin{gathered}
\mathbf{H}=\sigma^{2}\left(\mathbf{I}_{N-2 M} \otimes\left[\begin{array}{c}
\left.\left.\mathbf{I}_{M+1}\right]^{T}\right) \\
\mathbf{L}
\end{array}\right]\right. \\
\cdot\left[\begin{array}{c}
\mathbf{K}_{2 M+1,0} \\
\vdots \\
\mathbf{K}_{2 M+1,2 M} \\
\mathbf{0}_{(N-4 M-1)(2 M+1) \times(2 M+1)}
\end{array}\right]\left[\begin{array}{c}
\mathbf{I}_{M+1} \\
\mathbf{L}
\end{array}\right] .
\end{gathered}
$$

Substituting (17) into (12) gives (9).

\section{REFERENCES}

[1] D. C. Rife and R. R. Boorstyn, "Multiple tone parameter estimation from discrete-time observations," Bell Syst. Tech. J., pp. 1389-1410, Nov. 1976.

[2] P. Stoica and A. Nehorai, "Statistical analysis of two nonlinear leastsquares estimators of sine wave parameters in the colored noise case," in Proc. Int. Conf. Acoust., Speech, Signal Processing, vol. 4, Apr. 1988, pp. $2408-2411$

[3] V. F. Pisarenko, "The retrieval of harmonics by linear prediction," Geophys. J. R. Astron. Soc., vol. 33, pp. 347-366, 1973.

[4] R. O. Schmidt, "Multiple emitter location and signal parameter estimation," IEEE Trans. Antennas Propagat., vol. 34, pp. 276-280, Mar. 1986.

[5] R. Roy and T. Kailath, "ESPRIT-Estimation of signal parameter via rotational invariance techniques," IEEE Trans. Acoust., Speech, Signal Processing, vol. 37, pp. 984-995, July 1989.

[6] H. Li, P. Stoica, and J. Li, "Computationally efficient parameter estimation for harmonic sinusoidal signals," Signal Process., vol. 80, pp. 1937-1944, 2000.

[7] M. Zeytinoglu and K. M. Wong, "Detection of harmonic sets," IEEE Trans. Signal Processing, vol. 43, pp. 2618-2630, Nov. 1995.

[8] R. J. Mcaulay and T. F. Quateri, "Speech analysis/synthesis based on a sinusoidal representation," IEEE Trans. Acoust., Speech, Signal Processing, vol. 34, pp. 744-754, Apr. 1986.

[9] T. Nakatani and M. Miyoshi, "Blind dereverberation of single channel speech signal based on harmonic structure," in Proc. IEEE Int. Conf. Acoust. Speech, Signal Processing, vol. 1, Hong Kong, China, Apr. 2003, pp. $92-95$.

[10] L. Y. Ngan, Y. Wu, H. C. So, P. C. Ching, and S. W. Lee, "Joint time delay and pitch estimation for speaker localization," in Proc. IEEE Int. Symp. Circuits Systems, vol. 3, Bangkok, Thailand, May 2003, pp. 722-725.

[11] U. Kiencke and L. Nielsen, Automotive Control Systems for Engine, Driveline, and Vehicle. Berlin, Germany: Springer-Verlag, 2000.

[12] G. Simon, R. Pintelon, L. Sujbert, and J. Schoukens, "An efficient nonlinear least square multisine fitting algorithm," IEEE Trans. Instrum. Meas., vol. 45, pp. 750-755, Aug. 2002.

[13] T. Soderstrom and P. Stoica, System Identification. Englewood Cliffs, NJ: Prentice-Hall, 1989.

[14] H. Akaike, "A new look at the statistical model identification," IEEE Trans. Automat. Contr., vol. 19, pp. 716-723, Dec. 1974.

[15] J. Rissanen, "Modeling by shortest data description," Automatica, vol. 14, pp. 465-471, May 1978.

[16] S. Bakamidis, M. Dendrinos, and G. Carayannis, "SVD analysis by synthesis of harmonic signals," IEEE Trans. Signal Processing, vol. 39, pp. 472-477, Feb. 1991.

[17] J. Q. Zhang, S. J. Ovaska, and X. Z. Gao, "An eigenvalue residuum-based criterion for detection of the number of sinusoids in white Gaussian noise," in Proc. IEEE Southeastcon, Mar. 1999, pp. $154-158$

[18] Y. T. Chan, J. M. M. Lavoie, and J. B. Plant, "A parameter estimation approach to estimation of frequencies of sinusoids," IEEE Trans. Acoust., Speech, Signal Processing, vol. 29, pp. 214-219, Apr. 1981.

[19] T. J. Rivlin, Chebyshev Polynomials: From Approximation Theory to Algebra and Number Theory. New York: Wiley, 1990.

[20] V. U. Reddy, B. Egardt, and T. Kailath, "Least square type algorithm for adaptive implementation of Pisarenko's harmonic retrieval method," IEEE Trans. Acoust., Speech, Signal Processing, vol. 30, pp. 399-405, June 1982.

[21] P. Stoica and A. Eriksson, "MUSIC estimation of real-valued sine-wave frequencies," Signal Process., vol. 42, pp. 139-146, 1995.

[22] P. Stoica, P. Handel, and T. Soderstrom, "Approximate maximum likelihood frequency estimation," Automatica, vol. 30, no. 1, pp. 131-145, 1994.

[23] T. Brown and M. M. Wang, "An iterative algorithm for single-frequency estimation," IEEE Trans. Signal Processing, vol. 50, pp. 2671-2682, Nov. 2002 